\title{
Effect of extensional cyclic strain on the mechanical and physico-mechanical properties of PVC-NBR/graphite composites
}

\author{
S. A. Mansour* \\ Physics Department, Faculty of Science, Suez Canal University, Ismailia, Egypt \\ Received 13 September 2008; accepted in revised form 27 October 2008
}

\begin{abstract}
The variation of electrical resistivity as will as the mechanical properties of PVC (polyvinylchloride)-NBR (acrylonitrile butadiene rubber) based conductive composites filled with different concentrations of graphite were studied. These samples were studied as function of the constant deformation fatigue test. When the specimen was subjected to a large number of rapidly repeating strain cycles, and different strain amplitudes, the conductivity, $\sigma(T)$, shows an initial rapid fall followed by dynamic equilibrium. Increasing the number of cycles and strain amplitudes, the conductivity remains almost constant over the temperature range $30-140^{\circ} \mathrm{C}$. The equilibrium state between destruction and reconstruction of graphite particles has been detected for all strains of certain values of strain cycles $(1000,2000,3000$, and 4000 cycles for $30 \%$ strain amplitude). A preliminary study was done to optimize the possibility to use Conductive Polymer Composites (CPC) as a strain sensor and to evaluate its performance by an intrinsic physico-mechanical modification measurement. The electromechanical characterization was performed to demonstrate the adaptability and the correct functioning of the sensor as a strain gauge on the fabric. The coefficient of strain sensitivity $(K)$ was measured for 50 phr graphite/PVCNBR vulcanized at 3000 number of strain cycles and $30 \%$ strain amplitude. There was a broad maximum of $K$, with a peak value of 82 , which was much higher, compared to conventional wire resistors. A slight hysteresis was observed at unloading due to plasticity of the matrix. A good correlation exists between mechanical and electrical response to the strain sensitivity. Mechanical reinforcement was in accordance with the Quemada equation [1] and Guth model [2] attested to good particle-matrix adhesion. It was found that the viscous component of deformation gradually disappeared and the hardening occurred with increasing strain cycles. The modulus, fracture strength, and elongation at break increased with increasing filler volume fraction up to $40 \mathrm{phr}$ of graphite particles.
\end{abstract}

Keywords: polymer composites, mechanical properties, fracture and fatigue, modeling and simulation

\section{Introduction}

If there is a simple relationship between electrical conductivity and external variables such as temperature [3, 4] and mechanical stretching [5, 6], the composite has potential applications as a sensor. For these reasons, the development of new conductive composites requires an understanding of the changes in conductivity that might occur in service. Conversely, measurement of electrical conductivity under strain can be a probe to understanding microstructural changes. Carbon black-filled conductive rubbers have wide applications, such as pressure sensitive sensors which can be used for shock proof switches [7], sensors for measurement of vehicle weights to collect toll tax on roads [8], and smart flexible sensors adapted to textile structures, able to measure their strain deformations [9], and tactile sensor that is thin and flexible and able to attach to a curved surface and will make the robot operate in unstructured environments $[10$,

*Corresponding author, e-mail: saidhsa@yahoo.com

(C) BME-PT and GTE 
11]. For an electronic application of the CPC, the sensor should have a good linear behavior (with the effect of elongation), while the specific electrical resistivity of the system should be in a measurable range $(<100 \Omega \cdot \mathrm{m})[12]$.

Sau et al. [13] have reported that the electrical resistivity of strained samples from EPDM, 50/50 NBR/EPDM blend and NBR rubbers, depends on strain amplitude (\% elongation), frequency of stress-strain cycle, and also number of stress-strain cycles. Pramanik et al. [14] reported the electrical and mechanical properties of Short Carbon Fibres (SCF) and carbon-black-filled nitrile-rubber composites with the variation of filler concentration, filler blend composition, processing parameters, and compressive pressure have a pronounced influence on the conductivity of such composites. In the present work the composite materials composed of different amounts randomly dispersed of graphite particles in an insulating polymer matrix was examined aiming to attain a pronounced change in resistivity with prestrain. The PVC/NBR blend polymer was chosen as the matrix because of its environmental stability and ease in processing, graphite particles were also chosen for their availability and low resistivity, to form strain-sensitive composites.

A preliminary study was done to optimize the possibility to use CPC as a strain sensor and to evaluate its performance by an intrinsic physico-mechanical modification measurement. In this respect three factors are considered: 1 - the strain amplitude, 2 the number of cycles, and 3 - the optimal filler concentration from the percolation threshold. The results of this initial phase were aiming to have a good compromise between sensor sensitivity and resistivity value.

\section{Experimental}

\subsection{Materials and preparation of composites}

NBR (density $0.98 \mathrm{~g} / \mathrm{cm}^{3}$ and acrylonitrile content $34 \%$ ) and suspension polymerized PVC in powder form (density $=1.38 \mathrm{~g} / \mathrm{cm}^{3}$ ), was supplied by the Transport and Engineering Company (TRENCO), Alexandria, Egypt. Graphite (particle size diameter $<50 \mu \mathrm{m}$ and density $1.4 \mathrm{~g} / \mathrm{cm}^{3}$ ) were used as conductive and reinforcing filler. The compound formulations are presented in Table 1. The mixing was
Table 1. The formulations of graphite/PVC-NBR composites

\begin{tabular}{|c|c|}
\hline NBR & 60 \\
\hline PVC & 40 \\
\hline Graphite & X \\
\hline$* *$ DOP) & 50 \\
\hline$*$ Zinc oxide & 5 \\
\hline *Stearic acid & 2 \\
\hline *MBTS & 1.5 \\
\hline *TMTD & 0.5 \\
\hline "PBN & 1.0 \\
\hline *Sulfur & 2 \\
\hline
\end{tabular}

*means all ingredients in (phr) relative to NBR

**means the weight of DOP equals $50 \%$ of the weight of PVC

$\mathrm{X}$ is the amount of graphite which was varied from $0-70 \mathrm{phr}$ (parts per hundred rubber, by weight) relative to compound

accomplished in a home made two-roll mixing mill (length $0.3 \mathrm{~m}$, radius $0.15 \mathrm{~m}$, speed of slow roll $18 \mathrm{rpm}$ and gear ratio 1.4) was used. The mixing occurred for $40 \mathrm{~min}$ at a temperature of $25^{\circ} \mathrm{C}$, with same sequence of mixing of all compounding ingredients to avoid the effect of processing on electrical conductivity. The vulcanization was conducted under a heating press (KARL KOLB, Germany). Compression molded plaques were prepared by sandwiching the polymer between Mylar sheets, heating at $150 \pm 2^{\circ} \mathrm{C}$ for 30 min under minimal pressure $0.40 \mathrm{MPa}$. Plaques were rapidly cooled to ambient temperature. The vulcanized samples were shelf aged for $48 \mathrm{~h}$ before testing. The mixing time and vulcanization conditions were fixed for all samples.

\subsection{Measurement of the initial electrical properties without strain}

The electrical resistivities of different composites were measured with linear four point-probe method [15]. Also to diminish the contact resistance between the electrodes and the specimens, the four points were stuck with silver ink. The standard size adopted for each specimen was $40 \times 10 \times 2 \mathrm{~mm}^{3}$. Each resistance measurement was made from the slope of the voltage-current graph over the range of $0.2-10 \mathrm{~V}$. The current flow through the specimen was measured with a sensitive digital electrometer (616 Keithley, USA); the voltage was measured with DC power supply HY 5003. Before taking measurements, each sample was heated at $70^{\circ} \mathrm{C}$ for about one hour to remove absorbed moisture. 


\subsection{Application of pre-strain}

Some of the composite samples were subjected to a constant number (100) of rapid dynamic flex cycles of constant frequency ( 5.9 cycles/s), and at different amplitudes in a constant deformation fatigue tester [16]. The number of flex cycles and amplitude of flexing were varied. It was then subjected to different numbers of strain cycles with constant amplitude, which could be changed throughout the experiment. The rectangular specimen of $10 \times 40 \times$ $2 \mathrm{~mm}^{3}$ dimensions was clamped at both ends in a holder attached to the machine. The electrical properties of original (unstrained) and strained (under different conditions) samples were measured using the device described above. A regulated electrical oven was used to control the sample temperature to an accuracy of $\pm 1^{\circ} \mathrm{C}$ over a range of temperature from 30 to $140^{\circ} \mathrm{C}$.

\subsection{Mechanical techniques}

True stress and strain at rupture were carried out at room temperature on a tensile testing machine (AMETEK, USA). The test samples were strips of $2 \mathrm{~cm}$ working length and of $\sim 4 \mathrm{~mm}^{2}$ cross-sectional area. A digital force gauge (Hunter Spring ACCU Force II, $0.01 \mathrm{~N}$ resolution, USA) connected to a microprocessor was used to measure extension force $(F)$. A home made motor attachment was used to control the strain rate through a gearbox. The strain rate was preset using a variable DC power supply, and was measured using a microswitch attached to the apparatus wheel. The accuracy of strain measurement was about $0.1 \mathrm{~mm}$. The strain rate $\varepsilon$ throughout the experiment was fixed at $1 \mathrm{~s}^{-1}$. True stress and strains were calculated as shown by Equations (1) and (2) respectively:

True stess $=\frac{F}{A_{0}}(1+\varepsilon)$

$$
\text { Truestrain }=\ln (1+\varepsilon)
$$

where $\varepsilon=\Delta l / l_{0}$ is the tensile strain, and $A_{0}$ is the original cross-sectional area.

\subsection{Electrical properties with strain}

The resistivity was measured with a four-terminal technique [16] using a DC power supply model HY

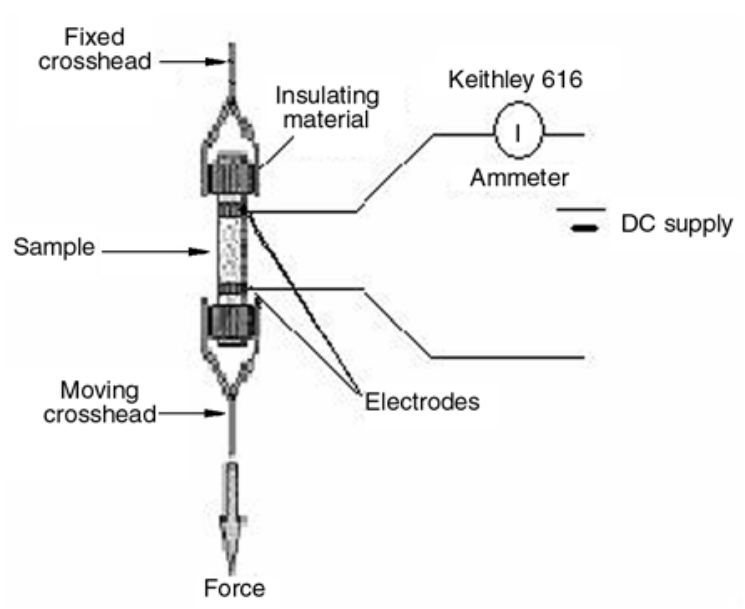

Figure 1. Schematics of DC electrical measurements during uniaxial extension

5003 as voltage source and a Keithley model 616 for current measurements. Specimens $(20 \times 2 \times$ 2) $\mathrm{mm}$ were uniaxially extended at room temperature using an (AMETEK, USA) universal testing machine. A constant crosshead speed was maintained to give a specified deformation rate. All the samples were only extended up to $50 \%$ elongation to avoid tensile failure of the specimens. The current and voltage electrodes were located $3 \mathrm{~mm}$ apart in the clamp and they were insulated from the machine frame (Figure 1). Conductive silver paint was applied between the sample surface and electrode to ensure a better electrical contact between them. The specimen's extension was measured from the displacement of the machine head. Assuming the sample volume remains constant (i.e., $\Delta V=0$ or $V=V_{0}$ ) throughout the experiment. The resistivity could be given by Equation (3):

$\rho=\frac{R V_{0}}{l_{0}^{2}(1+\varepsilon)^{2}}$

where $l_{0}$ is the initial length of the unstrained sample.

\section{Results and discussion}

\subsection{Electrical properties}

\subsubsection{Initial electrical properties without strain}

Figure 2 shows the variation of the resistivity with the volume fraction of filler. A dramatic decrease in resistivity, or threshold, is observed for given conductive filler content. This critical concentration corresponds to the percolation volume. At this 


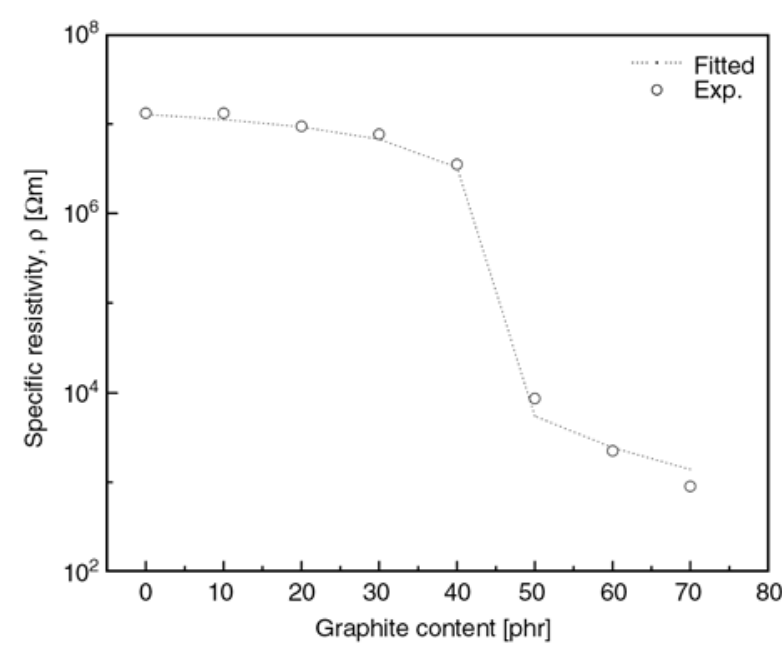

Figure 2. Resistivity as a function of graphite content. The dotted line is the statistical percolation fit.

point, the electric charges form electro-conductive channels, and there is a transition of the material from electrically insulating to conductive. This phenomenon is described in the percolation theory [17]. When the conductive particle concentration increases, the number of ohmic conductive paths increases, and the I-V relationship becomes more linear. The model that is most often used to quantify the changes in the transition and conductive regions is the so-called statistical percolation model. Proposed by Kirkpatrick [18] and Zallen [19], this model predicts the electrical resistivity of an insulator-conductor binary mixture by assuming random positions of the filler particles. The result is a power-law variation of the resistivity $\rho$, above the percolation threshold, as shown by Equation (4):

$\rho \alpha\left(\frac{\Phi-\Phi_{c}}{1-\Phi_{c}}\right)^{-t}$

where $\Phi$ is the volume fraction of filler, $\Phi_{c}$ the percolation threshold and $t$ is a universal exponent that is close to 2 for a 3D dispersion [20]. The twoparameter fit is represented in Figure 2 by the solid line. On the basis of this result, composite with $50 \mathrm{phr}$ filler was used to study the relationships between resistivity and mechanical deformation.

\subsubsection{Effects of the number of dynamic strain cycles and strain amplitude on conductivity}

In this paper, composites are identified by an alphanumeric system. The first letter represents the conductive graphite used in the 60/40 of an NBRPVC blend; the number indicates the loading of conductive graphite. For example, G50 represents a $60 / 40$ of an NBR-PVC blend containing $50 \mathrm{phr}$ of conductive graphite.

Figures 3 and 4 illustrate the effect of the number of strain cycles, and different strain amplitudes on the electrical conductivity of PVC-NBR blend system loaded with 30,50 , and $70 \mathrm{phr}$ graphite at room temperature. It is found that the sample with $50 \mathrm{phr}$ is more sensitive to both number of dynamic strain cycles and strain amplitude. The rapid change in conductivity here is presumably because it is very near to the critical percolation limit. Even small changes in the network structure would have the greatest effect on conductivity in the percolation region. Materials in this region are much more sensitive to strain than are more conductive com-

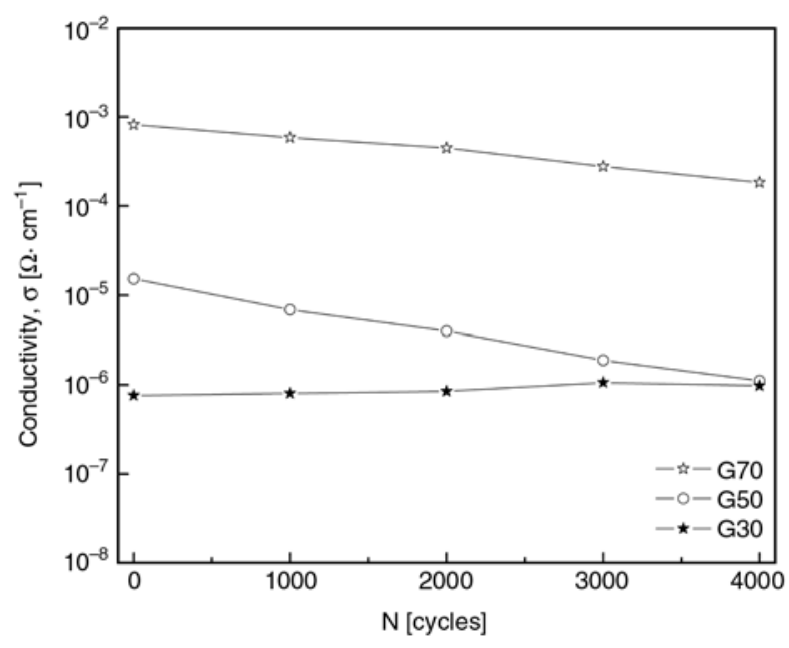

Figure 3. Dependence of the electrical conductivity $(\sigma)$ on the number of strain cycles $(N)$ at constant amplitude of $30 \%$

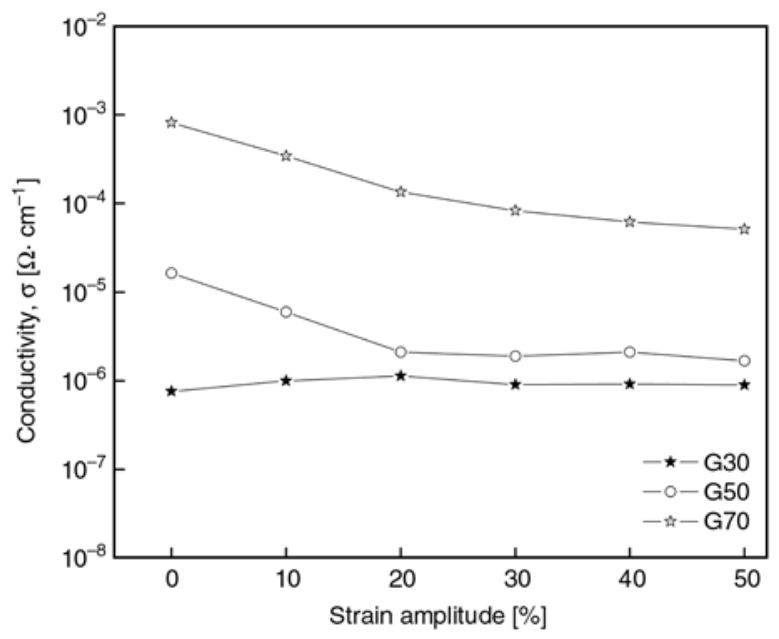

Figure 4. Dependence of the electrical conductivity $(\sigma)$ on strain amplitude after 100 strain cycles 
pounds and then this is the reason behind the choice to study the effect of dynamic strain on the electrical properties of $50 \mathrm{phr}$ graphite/PVC-NBR vulcanized.

High structure graphite manifests a natural tendency towards breakdown. Destruction and reconstruction processes may also occur by mechanical deformation and by thermal expansion and contraction processes. It is conventional to distinguish between low strain and high strain amplitudes and their influence on the properties of rubber composites. Strains below $30 \%$ are considered low, while those above $30 \%$ are considered high [21]. At low strain amplitudes $(<20 \%)$ for G30 sample, the formation of graphite aggregates predominates over their destruction, which in turn slightly increase the initial conductivity of the sample. But for both samples (G50, and G70) the initial conductivity decreases with increasing amplitude of cyclic strain. It could be explained as follows: during the initial strain amplitude, the breakdown of the conductive network becomes more predominant, and at higher frequency level (at strain amplitudes $>20 \%$, for all samples), the rate of formation of conductive network becomes faster and the formation and breakdown processes balance each other, leading to a plateau in the plots of conductivity.

\subsubsection{Effects of the number of dynamic strain cycles and cyclic strain amplitude on the electrical conductivity $\sigma(T)$ filled with $50 \mathrm{phr}$ of graphite}

In principle, composites whose resistivities sharply vary near a critical percolation threshold may show a notable change in the resistivity with the temperature. Consequently, it is only possible to find one composite from the PVC-NBR/graphite series for examining the dependence of conductivity on temperature with the effect of repeated cyclic strains at constant amplitude (30\%), and strain amplitude (at constant number of cycles 100) for 50 graphite/ PVC-NBR blend.

Figures 5 and 6 present plots of conductivity against the temperature, which can be explained as follows:

(i) The number of strain cycles (0, 1000 and 2000) leads to the initial breakage of the existing continuous conducting network. The increase in temperature leads to an increase in the gap

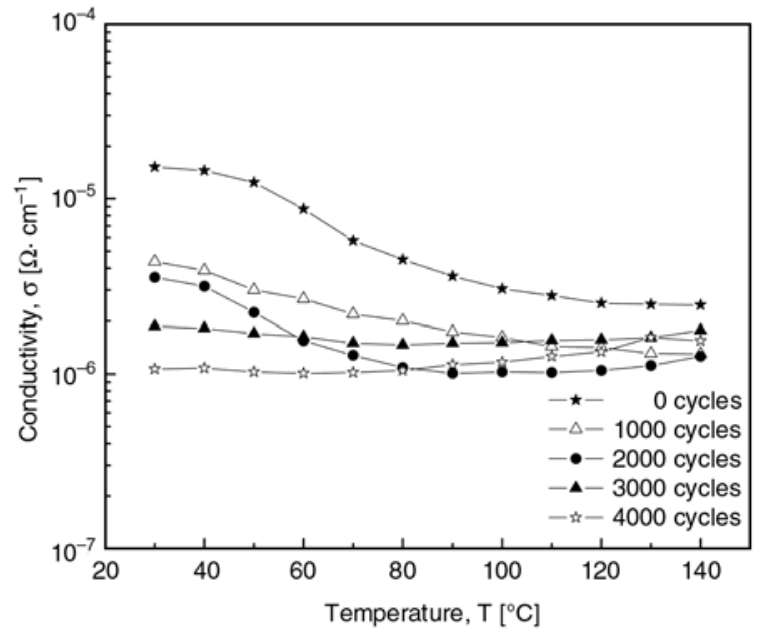

Figure 5. Temperature dependence of the electrical conductivity $(\sigma)$ of 50 graphite/PVC-NBR on different numbers of strain cycles (at constant strain amplitude 30\%)

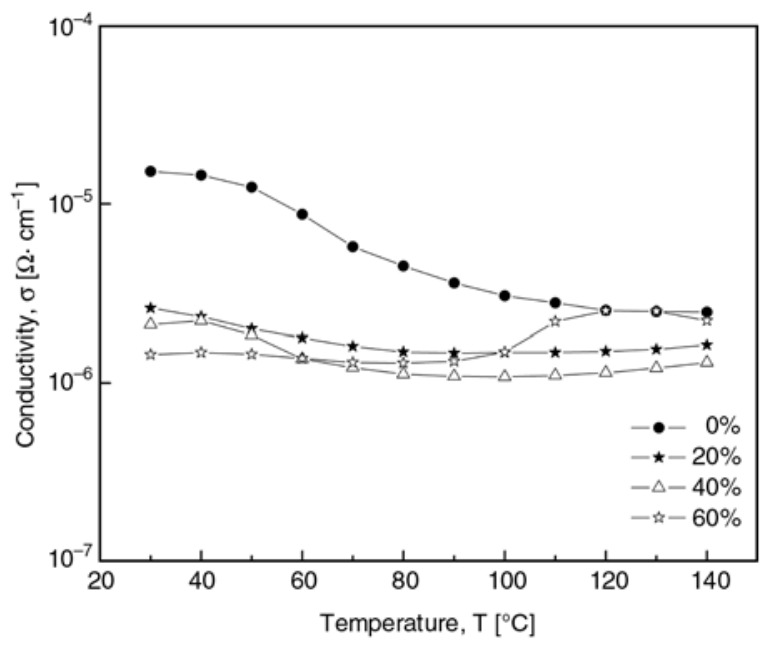

Figure 6. Temperature dependence of the electrical conductivity $(\sigma)$ of 50 graphite/PVC-NBR sample at different strain amplitude (at constant number of cycles 100)

between graphite structure and the masking of graphite particles by insulating rubber layers, that results in a slight decrease in conductivity.

(ii) After this breakdown process, the blend shows a greater tendency for alignment of graphite during the application of cyclic dynamic strain.

(iii) At high numbers of strain cycles (3000 and $4000)$ and high degree strain amplitude (20, 40 and $60 \%$ ) the almost temperature independent behaviour of the electrical conductivity of the blend could be due to a balance between the two mechanisms of conduction mentioned above and to direct contact between graphite aggregates; i.e. the high degree of orientation 
of graphite aggregates may nullify the effect of chain breakage and thus a plateau effect is observed. So that it is interesting to study the effect of frequency of stress-strain cycling on electrical resistivity of G50 at strain amplitude of $30 \%$ and after 3000 cycles.

\subsection{Mechanical properties}

The mechanical performance of the PVC-NBR/ graphite composites was studied in terms of Young's modulus, tensile strength and elongation at break. It is well known that the increase in modulus is due to strong interactions between polymer chains and particles and/or between particles and particles. Figure 7 illustrates the dependence of elastic modulus $(E)$ on graphite volume fraction. It is shown that the elastic modulus $(E)$ increases with increasing graphite content. For composites filled with low amounts of weakly interacting spherical particles, the behavior of the change of the elastic modulus depends on the volume fraction of fillers $(\Phi)$, and may be predicted by Quemada Equation (5) [1]:

$$
E=\frac{E_{m}}{(1-0.5 N \Phi)^{2}}
$$

where $E_{m}$ is the modulus of the elastomer matrix, $\mathrm{N}$ is variable coefficient (usually $N=2.5$ ). The theoretical curve corresponding to Equation (5) shows good accordance with the experimental data as shown in Figure 7.

The results were also compared with Guth reinforcement model [2]. The model for reinforcing particles has the form as given by Equation (6):

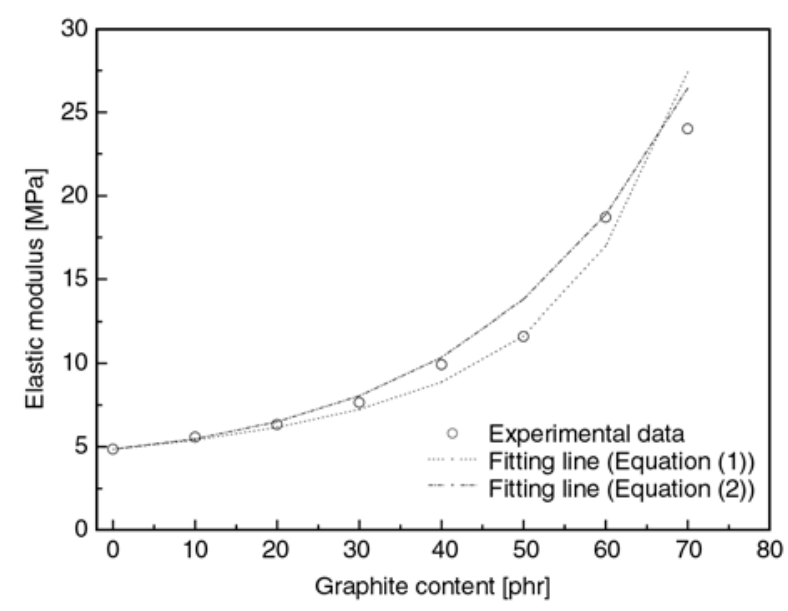

Figure 7. Elastic modulus $(E)$ plotted against graphite content [phr]
$E=E_{m}\left(1+2.5 \Phi+14.1 \Phi^{2}\right)$

Where the linear term accounts for the reinforcing effect of individual particles and the second power term is the contribution of particle pair interactions. This model significantly underestimates the observed reinforcement, as shown in Figure 7. The difference between experimental data and Equation (6) was even observed for a graphite content $40 \mathrm{phr}$, where the model is most applicable.

The variation of tensile strength is shown in Figure 8. It is observed that the tensile strength increases with increase in filler loading. This can be explained by the following mechanisms: tensile strength is related to the energy required to deform and fracture the polymer chains. The effective crosslink density of the composites increases with increase in filler loading attributed to the increase of polymer-filler interaction with the addition of filler. Thus, at low filler loading, the energy required to deform and fracture the polymer chains is less because of lower crosslink density, whereas at high filler loading, attributed to the higher crosslink density, the energy required to deform and fracture the polymer chains is high. The ability of polymer to stretch several times its original length is one of its chief characteristics. The variation of elongation at break with filler loading is presented in Figure 9.

It can be seen that elongation at break increases with increase in filler loading to a maximum and then shows a decrease with further loading of the filler. This is because at very low filler content, when the matrix is not sufficiently reinforced, it

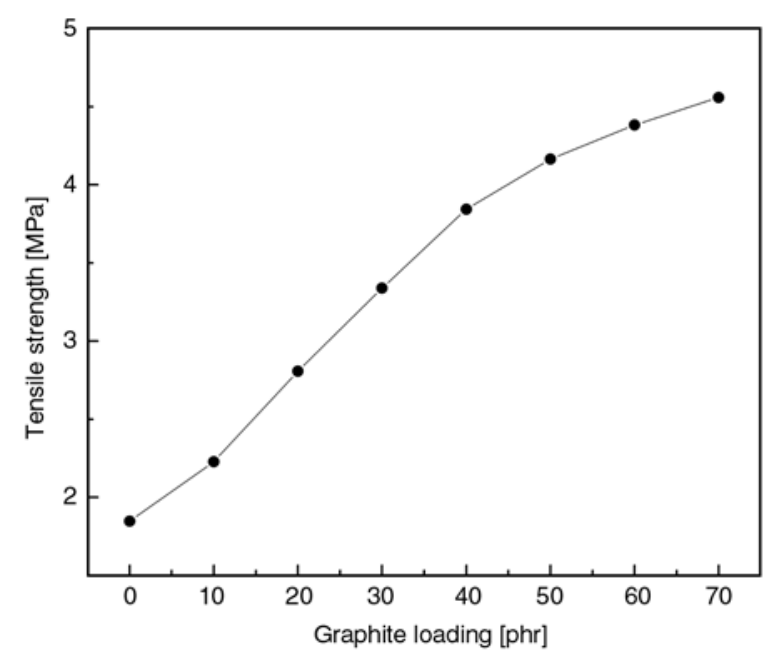

Figure 8. Effect of filler loading on tensile strength of PVC-NBR composites 


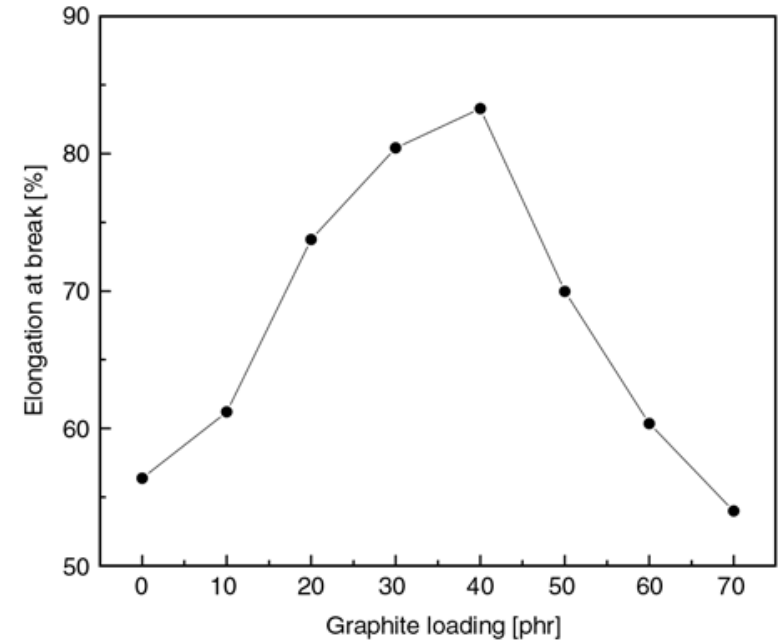

Figure 9. Effect of filler loading on elongation at break of PVC-NBR composites

cannot sustain the load and so failure occurs at lower elongation. However, with increase in filler loading, the matrix is progressively reinforced and a higher elongation at breaking point is observed. With further loading, the molecular segmental motion is restricted because of physical interaction between the filler aggregates and polymer chains. Consequently, the elongation at breaking point decreases with increasing filler loading.

The composites containing $50 \mathrm{phr}$ (G50-0 cycle, and G50-3000 cycles) at constant amplitude 30\%, display appreciable irreversibility in the stressstrain plots with applied strain during the extension-retraction cycle. The materials studied here have shown small tensile set as observed from Fig-

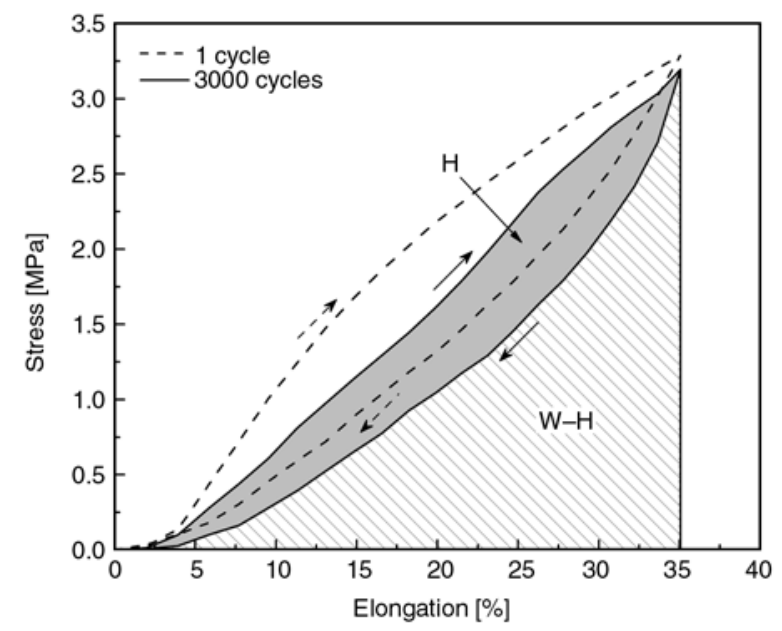

Figure 10. Stress-strain plots for the extension-retraction cycle of (G50-0 cycle, and G50-3000 cycles) composites. Hysteresis lost work $(H)$, and the work per one cycle $(W)$. ure 10. However, the presence of graphite particles in the composites reduces the set property which in turn reflects a better interaction between graphite and rubber matrix. The reduction in the hysteresis of G50-3000 cycles compared to G50-0 cycle was attributed to viscoelastic relaxation of the matrix. The fact that the stress-strain characteristic of this rubber causes a hysteresis effect was experimentally clarified by Shimojo et al. [22]. The index for showing the degree of hysteresis is the ratio $H / W$, where $H$ is the work lost due to hysteresis and $W$ is the work per cycle, as shown in Figure 10. The ratio $(H / W)$ was measured experimentally $(0.296$ for G50-0, and 0.301 for G50-3000 cycles). The results confirm that the ratio of $(H / W)$ does not change up to about 3000 cycles in frequency. As a result, it was confirmed that the form of the hysteresis loop for each cycle did not change, even as cycles were repeated.

The equilibrium stress-strain relation and number of cycles to failure for fatigue life of (3000 cycles) were illustrated in Figure 11. From this figure, the equilibrium stress-strain relation for the first strain cycle exhibits the significant nonlinear deformation and low maximum stress, and then this nonlinearity of deformation decreases and the maximum equilibrium stress increases with an increase in strain cycles. This means that the viscoplastic component of deformation gradually disappears and the hardening occurs during fatigue.

To characterize the variation of equilibrium stressstrain relation during fatigue, the Young's modulus $E_{N}$, and accumulated strain by fatigue ratcheting $\varepsilon_{N}$

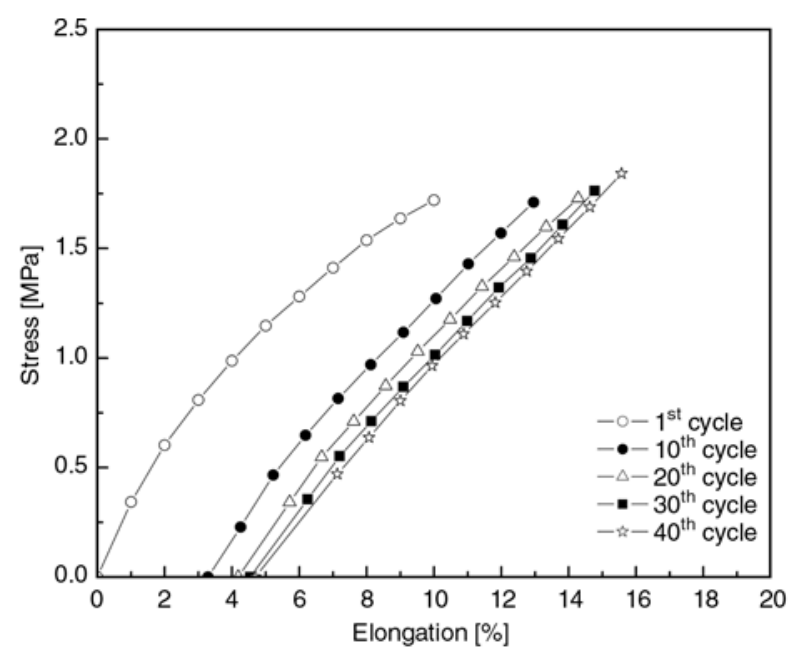

Figure 11. Variation of equilibrium stress-strain relation with fatigue (strain cycles for G50-3000 cycles) 


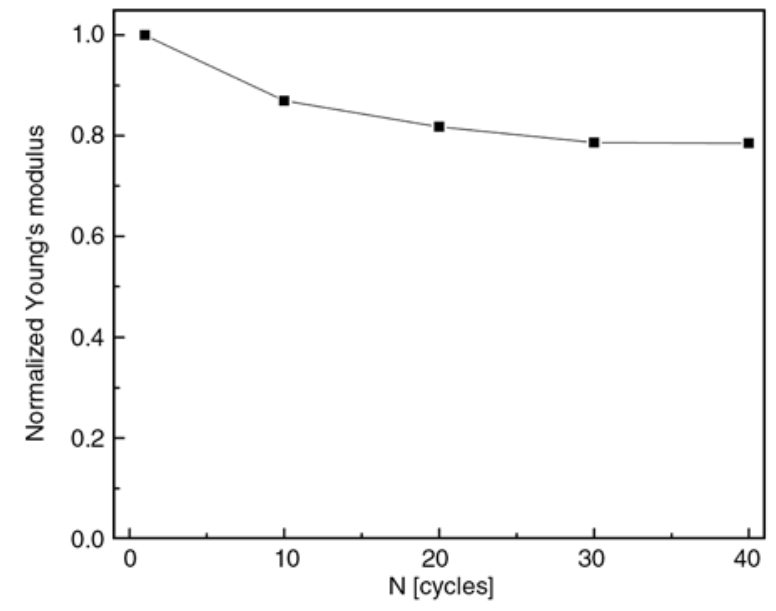

Figure 12. Variation of Young's modulus with the number of cycles for G50-3000 cycles

are determined from the equilibrium stress-strain relation for the $N^{\text {th }}$ strain cycle as shown in Figure 11. Then normalized Young's modulus $E_{N} / E_{1}$, and accumulated strain $\varepsilon_{N}$ is shown as functions of the number of strain cycles in Figure 12. The stiffness reduces significantly at the early 10 strain cycles and then becomes constant.

\subsection{Electromechanical properties}

Depending on the application, strain gauges can be either metal or semiconductor strain gauges. The latter ones are made of semiconductor materials, but have advantages and disadvantages. On the one hand, semiconductor strain gauges are very small and have large gauge factors. In fact, resistance changes here are much bigger than those obtained with metal strain gauges.

When the sample deform, the fraction of electrical resistance change $(\Delta R / R)$ is expressed by Equation (7) [23]:

$$
\frac{\Delta R}{R}=K \varepsilon
$$

where, $K$ is a gauge factor. The longitudinal extension of the sensor leads to a shrinking in the crosssection area $A$, and according to Equation (1), the resistance $R$ will increase. Moreover, an extension of the sensor length will cause a decrease in the number of electrical connections between the conductive particles in the CPC, and the resistance will thus increase. The combination of these two phenomena will therefore lead to an important increase

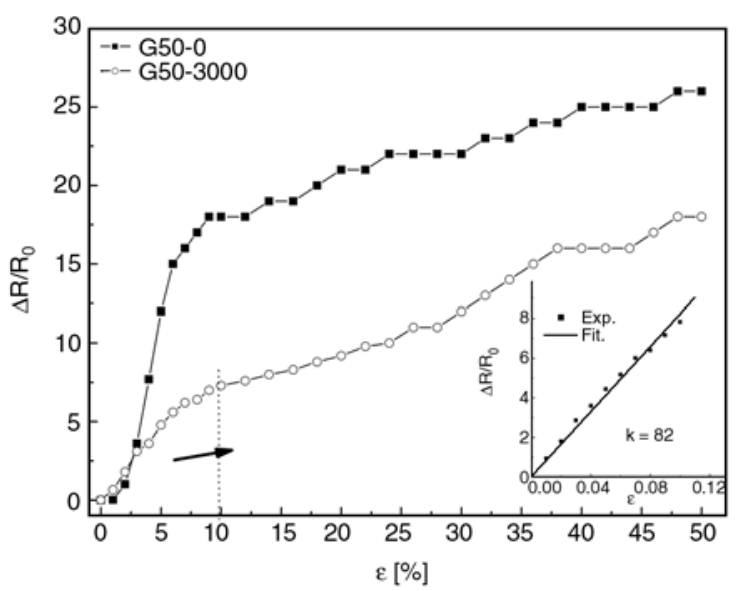

Figure 13. Dependence of relative change of resistance vs. strain of the sensor

of the resistance for a small strain deformation. Although the gauge factor varies depending on materials, the value is approximately around 2 4 for most of metallic materials and 80 170 or -95 to -110 for semiconductor strain gauges, depending on the doping level and the design [24, 25]. For classical metallic conductors (copper, nickel), this factor is 2.1 at the maximum. However, the strain range covered by a classical metal gauge is between 0.1 and $0.5 \%$ [26]. Figure 13 shows the dependence of relative change of resistance on strain over the range of elongation. The curve obtained on Figure 13 can be divided in two regions for the two samples G50-0 cycle, and G50-3000 cycles: the first one corresponds to a strain below $10 \%$, where the sensor response is practically linear. It is interesting that the strain sensitivity coefficient $K=$ $\Delta R / R_{0} \varepsilon$ (G50-0 cycles) can be very high, up to 203 , which is more than a decade higher compared to commercial wire strain gauges, while the values of $K$ is 82 , which is in agreement with semiconductor strain gages $[25,26]$. In the second region, for strain values greater than $10 \%, K$ was found to be most similar, and equal to 20 for two samples. A study demonstrated that the composite exhibited Ohmic behavior before and after stretching to $10 \%$ strain.

The normalized resistivity curves for G50-0, and G50-3000 with deformation to 5\% strain illustrated in Figure 14. The resistivity exhibited a small hysteresis loops. The decrease in resistivity upon unloading up to $2 \%$ strain was due to relaxation of the stretched polymer, which allowed the particles to reform the network. 


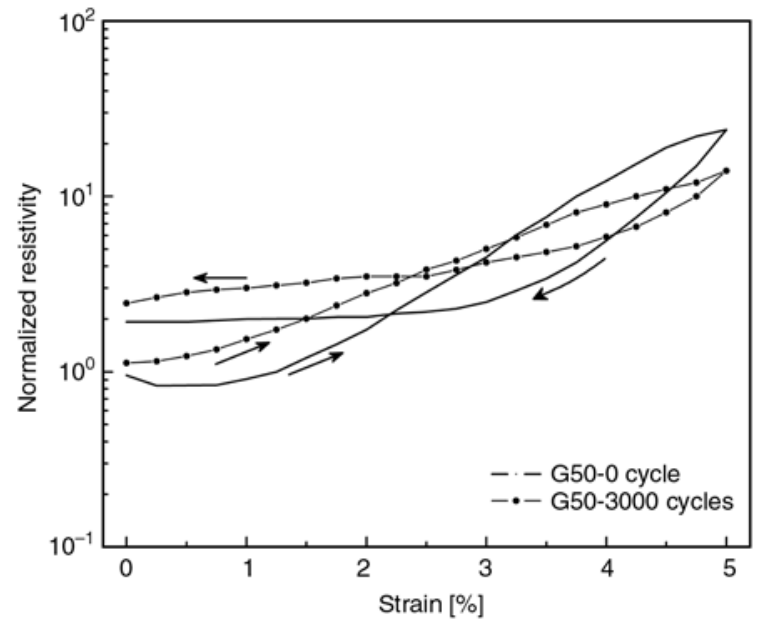

Figure 14. Effect of extension-retraction cycle to $5 \%$ strain on the normalized resistivity of a composite with graphite $50 \mathrm{phr}$. G50-0 cycle (solid lines), and G50-3000 cycles (circles)

This complex behavior demonstrates the dynamic and time-dependent nature of microstructural changes in the region of low strain. This region is generally attributed to network breakage; the composite exhibited no permanent strain or irreversible electrical properties during mechanical cycling. The disadvantage of these types of composites is the hysteretic behavior of the sample resistivity when extension-retraction is applied. Composites (G50 at strain amplitude of $30 \%$ and after 3000 number of cycles) have a flatter temperature response but unfortunately are not suitable for sensor application. Improvement of particle-polymer chemical interaction and choice of matrix may be able to overcome this problem in the future.

\section{Conclusions}

The percolation thresholds detected for composites were achieved through the applicability between the electrical as well as the mechanical properties. The composites demonstrated modulus reinforcement in accordance with the Guth model for good particle-matrix adhesion. Typically, reinforcement even with high-structure graphite is accompanied by a decrease in elongation to break. The disadvantage of composites with graphite did not maintain good mechanical properties, generally exhibited an increase in resistivity with strain, and exhibited irreversible changes in both mechanical and electrical properties after extension to even low strains. Cyclic strain markedly affects the value and behav- iour of conductivity with temperature, also is greatly changed by both strain amplitude and the number of strain cycles. The conductivity of $50 \mathrm{phr}$ graphite/PVC-NBR at amplitude of 30\% and after 3000 cycles becomes temperature independent. From the variation of the equilibrium stress-strain relations with strain cycles, it is found that the viscous component of deformation gradually disappears and the hardening occurs with increasing strain cycles. The filler loading in the composite which gave the best compromise between sensor sensitivity and measurable resistance values was found to be much higher than the concentration at the percolation threshold.

The electromechanical characterization showed that the sensor presented a classical ohmic linear behaviour for the lower strain levels $10 \%$, the average gauge factor $K$ in this range is 82 .

\section{References}

[1] Rusu M., Sofian N., Rusu D.: Mechanical and thermal properties of zinc powder filled high density polyethylene composites. Polymer Testing, 20, 409 (2001).

[2] Guth E.: Theory of filler reinforcement. Journal of Applied Physics, 16, 20-25 (1945).

[3] Flandin L., Hiltner A., Baer E.: Interrelationships between electrical and mechanical properties of a carbon black-filled ethylene-octene elastomer. Polymer, 42, 827-38 (2001).

[4] Shevchenko V. G., Ponomarenko A. T., Klason C.: Strain sensitive polymer composite material. Smart Material Structures, 4, 31-35 (1995).

[5] Flandin L., Chang A., Nazarenko S., Hiltner A., Baer E.: Effect of strain on the properties of an ethyleneoctene elastomer with conductive carbon fillers. Journal of Applied Polymer Science, 76, 894-905 (2000).

[6] Feller J-F., Linossier I., Levesque G.: Conductive polymers composites (CPCs): Comparison of electrical properties of poly (ethylene-co-ethyl acrylate)-carbon black with poly(butyleneterephthalate)/poly(ethylene-co-ethylacrylate)-carbon black. Polymer Advanced Technology, 13, 714-724 (2002).

[7] Krupa I., Novak I., Chodak I.: Electrically and thermally conductive polyethylene/graphite composites and their mechanical properties. Synthetic Metals, 145, 245-252 (2004).

[8] Norman R. H.: Conductive rubber and plastics. Elsevier, London (1970).

[9] Cochrane C., Koncar V., Lewandowski M., Dufour C.: Design and development of a flexible strain sensor for textile structures based on a conductive polymer composite. Sensors, 7, 473-492 (2007). 
[10] Shimojo M., Namiki A., Ishikawa M., Makino R., Mabuchi K.: A tactile sensor sheet using pressure conductive rubber with electrical-wires stitched method. IEEE Sensors Journal, 4, 589-596 (2004).

[11] Inaba M., Hoshino Y., Nagasaka K., Ninomiya T., Kagami S., Inoue H.: A full-body tactile sensor suit using electrically conductive fabric and strings. in 'Proceedings of the IEEE/RSJ International Confference of Intelligent Robots and Systems, Dayton, USA' vol 2, 450-457 (1996).

[12] Flandin L., Brechet Y., Cavaille J-Y.: Electrically conductive polymer nanocomposites as deformation sensors. Composites Science and Technology, 61, 895901 (2001).

[13] Sau K. P., Chaki T. K., Khastgir D.: The change in conductivity of a rubber-carbon black composite subjected to different modes of pre-strain. Composites Part A: Applied Science and Manufacturing, 29, 363-370 (1998).

[14] Pramanik P. K., Khastagir D., Saha T. N.: Effect of extensional strain on the resistivity of electrically conductive nitrile-rubber composites filled with carbon filler. Journal of Materials Science, 28, 3539-3546 (1993).

[15] Smits F. M.: Measurement of sheet resistivities with the four-point probe. Bell System Technical Journal, 37, 711-718 (1958).

[16] Amin M., Nasr G. M., Hassan H. H., El-Guiziri S., Abu-Abdeen M.: Investigation on the dependence of the electrical conductivity of FEF/SBR vulcanizates on the cyclic strain. Polymer Bulletin, 22, 413-420 (1989).
[17] Roldughin V. I., Vysotskii V. V.: Percolation properties of metal-filled polymer films, structure and mechanisms of conductivity. Progress in Organic Coating, 39, 81-100 (2000).

[18] Kirkpatrick S.: Percolation and conduction. Review Modern Physics, 45, 574-588 (1973).

[19] Zallen R.: The physics of amorphous solids. Wiley, New York (1983).

[20] Derrida B., Stauffer D., Herrmann H. J., Vannemius J.: Transfer matrix calculation of conductivity in three-dimensional random resistor networks at percolation threshold. Journal de Physique-Letters, 44, 701-706 (1983).

[21] Norman R. H.: Conductive rubbers and plastics. Elsevier, London (1970).

[22] Shimojo M., Ishikawa M., Kanaya K.: A flexible high resolution tactile imager with video signal output. in 'IEEE International Conference of Robotics and Automation, Sacramento, USA' vol 1, 384-391 (1991).

[23] Todoroki A., Yoshida J.: Electrical resistance change of unidirectional CFRP due to applied load. JSME International Journal, Series A, 47, 357-364 (2004).

[24] Shankland E. P.: Piezoresistive silicon pressure sensors. Sensors Magazine, 22, 22-26 (1991).

[25] Frank R.: Understanding smart sensors. Artech House, Norwood, USA (2000).

[26] Asch G.: Les capteurs en instrumentation industrielle. Dunod, Paris (2000). 